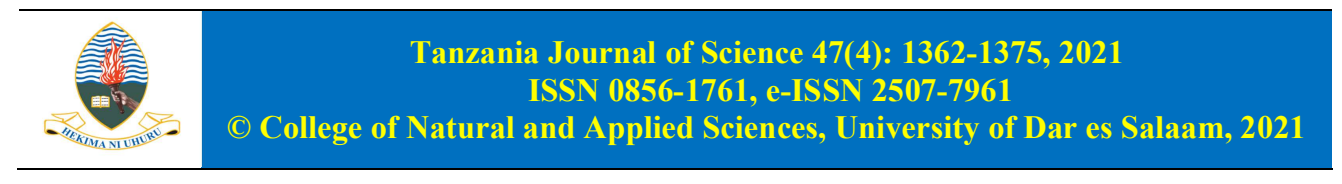

\title{
Pair Distribution Function Analysis and Electrochemical Performance of Mesoporous Carbon Nanomaterials Synthesized Through $\mathrm{KOH}$ and $\mathrm{ZnCl}_{2}$ Activation
}

\author{
Talam E. Kibona ${ }^{1 *}$, Sarah Frisco ${ }^{2}$ and Maxwell W. Terban ${ }^{3}$ \\ ${ }^{1}$ Department of Physics, Mkwawa University College, University of Dar es Salaam P. O. Box 2513 \\ Iringa,Tanzania. E-mail:kibonatalam@gmail.com,kibona@muce.ac.tz \\ ${ }^{2}$ Department of Materials Science and Engineering, Carnegie Mellon University, Pittsburgh, PA \\ 15213, United States. E-mail: friscosarah@gmail.com \\ ${ }^{3}$ Department of Applied Physics and Applied Mathematics, Columbia University, New York, NY \\ 10027, United States. E-mail: mwt2115@columbia.edu \\ *Corresponding author,
}

Received 21 Jun 2021, Revised 5 Oct 2021, Accepted 6 Oct 2021, Published Oct 2021

DOI: https://dx.doi.org/10.4314/tjs.v47i4.3

\begin{abstract}
Mesoporous carbon has been synthesized by activating carbonized biogas slurry residues with $\mathrm{ZnCl}_{2}$ and $\mathrm{KOH}$ simultaneously. The carbon to activating agent mass ratios were kept at 1:4, while the $\mathrm{ZnCl}_{2}$ to $\mathrm{KOH}$ mass ratio varied from 4:0, 3:1, 2:2, 1:3, to $0: 4$. The highest BET specific surface area of $361 \mathrm{~m}^{2} \mathrm{~g}^{-1}$, micropore surface area of $231 \mathrm{~m}^{2} \mathrm{~g}^{-1}$, mesopore surface area of $125 \mathrm{~m}^{2}$ $\mathrm{g}^{-1}$, and total pore volume of $0.23 \mathrm{~cm}^{3} \mathrm{~g}^{-1}$ which amounted to $78 \%$ mesopore content, were obtained for the sample with $3: 1 \mathrm{ZnCl}_{2}$ to $\mathrm{KOH}$ mass ratio. Scanning electron microscope (SEM) images were acquired to determine the surface morphology and energy dispersive X-ray (EDX) was used to determine surface composition of the samples. The short, medium, and long-range orders of the synthesized materials were studied using pair distribution function (PDF) analysis. PDF showed that in addition to the locally ordered carbon and silica phase components, samples activated using combined $\mathrm{ZnCl}_{2}$ and $\mathrm{KOH}$ also contained crystalline $\mathrm{Zn}_{2} \mathrm{SiO}_{4}$ phase with the willemite structure. Electrochemical studies in three-electrode cell system revealed maximum specific capacitance of $216 \mathrm{~F} \mathrm{~g}^{-1}$ exhibited by sample with a $\mathrm{ZnCl}_{2}$ : $\mathrm{KOH}$ mass ratio of $3: 1$ at a scan rate of $5 \mathrm{mV} \mathrm{s}^{-1}$.
\end{abstract}

Keywords: Mesoporous carbon; Pair distribution function, Specific capacitance, supercapacitor.

\section{Introduction}

High power delivery of the stored energy devices is deemed to be the best fit for intermittent renewable energy sources, portable electronics, and braking in transport for energy recovery systems. The storage can be achieved through supercapacitors due to their high power density (Castro-Gutiérrez et al. 2021). However, the energy density of supercapacitors is low and needs improvement. Energy storage in supercapacitors is facilitated by the porous nature and surface area of the electrodes, which can be made through chemical activation of carbonaceous materials derived from biomass (Enock et al. 2017, Wang et al. 2020). Activated carbon can be synthesized by direct carbonization of a biomass precursor, followed by chemical activation using $\mathrm{KOH}$ (Kibona 2019, Wang et al. 2020), $\mathrm{NaOH}$ (Baig and Gul 2021), phosphoric acid (Elisadiki et al. 2019, $\mathrm{Hu}$ et al. 2021) and $\mathrm{ZnCl}_{2}$ (Lin et al. 2020) as activating agents. Potassium hydroxide $(\mathrm{KOH})$

1362 
has an advantage of producing activated carbon at low activating temperatures with high surface area and well-defined pore distribution. It also increases the surface oxygen of the porous carbon, which is important for pseudocapacitance and wettability (Ma et al. 2013, Wang et al. 2020). $\mathrm{ZnCl}_{2}$ can be used in molten salt synthesis of activated carbons from biomass (Shang et al. 2015). It has the advantages of dehydrating the biomass, increases the yield of activated carbon, and restricting the formation of tar, thus promoting decomposition during carbonization. When $\mathrm{ZnCl}_{2}$ is used in molten salt synthesis route, the carbonization temperature decreases, this is because $\mathrm{ZnCl}_{2}$ favours a flux environment, which allows for solid-phase reactions. Therefore, in order to improve the microstructure of the carbon, combined chemical activations and molten salt synthesis routes are important. An attempt was performed by mixing the formaldehyde/phenol based nanofibre paper with $\mathrm{KOH}$, sodium fluoride $(\mathrm{NaF})$, sodium chloride $(\mathrm{NaCl})$ in the weight ratio of $1(\mathrm{KOH}): 1(\mathrm{NaF}): 19(\mathrm{NaCl})$ (Ma et al. 2017). It was observed that the mixture of $\mathrm{KOH}, \mathrm{NaF}$, and $\mathrm{NaCl}$ had a significant effect on the specific surface area, which increased from $449 \mathrm{~m}^{2} \mathrm{~g}^{-1}$ for samples without molten salts to $1007 \mathrm{~m}^{2} \mathrm{~g}^{-1}$ when molten salts were combined with $\mathrm{KOH}$.

It has been proven that mesoporous carbon materials can be synthesized by activating biomass with $\mathrm{KOH}$ at different $\mathrm{KOH}$ to carbon ratios (Enock et al. 2017, Cao et al. 2020, Castro-Gutiérrez et al. 2021). In this study, both $\mathrm{KOH}$ and $\mathrm{ZnCl}_{2}$ were used together in synthesizing active materials from disposed biogas wastes. The effectiveness of using $\mathrm{ZnCl}_{2}$ and $\mathrm{KOH}$ as molten salt and activating agent when used simultaneously in preparing the activated materials was investigated. The resulting materials were heterogeneous mixtures of both short and long-range ordered phases. Pair distribution function (PDF) analysis was thus used to simultaneously characterize the order of the materials, using the information from both the Bragg and diffuse scattering components in the X-ray diffraction (XRD) patterns.

\section{Materials and Methods}

Synthesis of porous carbon

Cow dung fed biogas slurry was used as the biomass source in this study. The slurry was collected from the disposed waste at biogas plant in Arusha Tanzania. The activated materials were synthesized as follows: The biogas wastes were dried in air for 14 days then ground in a high-speed rotary cutting mill to form powder. The powder ( $3 \mathrm{~g})$ was carbonized in a horizontal tube furnace at $550{ }^{\circ} \mathrm{C}$ for $1 \mathrm{~h}$. The resulting carbonized char was mixed with $\mathrm{ZnCl}_{2}$ and $\mathrm{KOH}$ then activated at $800{ }^{\circ} \mathrm{C}$ for 1 $\mathrm{h}$. The carbonization and activation process was done at a heating rate of $10{ }^{\circ} \mathrm{C} / \mathrm{min}$ in the presence of white-spot nitrogen gas. The samples produced via this route were referred to as $\mathrm{BC}-\mathrm{X}-\mathrm{Y}$, where $\mathrm{X}$ represents the mass of $\mathrm{ZnCl}_{2}$, and $\mathrm{Y}$ represents the mass of $\mathrm{KOH}$, used in the synthesis. For comparison purposes, two samples were prepared by activating carbonized materials with $\mathrm{ZnCl}_{2}$ and $\mathrm{KOH}$ at $800{ }^{\circ} \mathrm{C}$ for $1 \mathrm{~h}$ separately. These samples were labelled as BC-4-0 and BC-0-4 for $\mathrm{ZnCl}_{2}$ and $\mathrm{KOH}$ activation, respectively. The unactivated sample was designated as BC-0-0. The activated materials were washed with $1 \mathrm{M} \mathrm{HCl}$ so as to remove residual inorganics. The samples were further washed with distilled water until the $\mathrm{pH}$ of the filtrate was around 7 when measured using $\mathrm{pH}$ meter (ORION STAR A214 Thermo Scientific). The washed samples were then dried in an oven at $120^{\circ} \mathrm{C}$ for $12 \mathrm{~h}$.

\section{Surface morphology and textural characterization}

The morphologies of the sample were investigated using Scanning Electron Microscopy (SEM-PHILIPS XLS 30 with secondary electron detector and acceleration voltage of $20 \mathrm{kV}$. The samples were coated with platinum before measurements were performed. Energy dispersive X-ray (EDX) microanalysis system attached to the SEM was 
used to investigate the surface composition of the samples. In order to characterise the pore morphology of the materials, Micromeritics ASAP 2020 was used to study the nitrogen adsorption and desorption of the materials at 77 $\mathrm{K}$. The BET and BJH methods were used to determine the specific surface area and pore distribution, respectively.

\section{Total scattering measurements and PDF analysis}

Synchrotron X-ray total scattering experiments were conducted on beamline 28ID-2 at the National Synchrotron Light Source II (NSLS-II) at Brookhaven National Laboratory (BNL). Samples were packed into 1 mm ID kapton capillaries and measured at 100 $\mathrm{K}$ using a flowing nitrogen Cryocooler. The rapid acquisition PDF (RaPDF) (Chupas et al. 2003) technique was used with an X-ray energy of $67.563 \mathrm{keV}(\lambda=0.18351 \AA)$. A large area 2D PerkinElmer detector $(2048 \times 2048$ pixels, $200 \times 200 \mu \mathrm{m}$ pixel size) was mounted orthogonal to the beam path with a sample-todetector distance of $205.4850 \mathrm{~mm}$. The setup was calibrated by measuring a standard sample of known lattice parameter (Ni). The raw 2D data were azimuthally integrated and converted to $1 \mathrm{D}$ intensity versus the magnitude of the scattering momentum transfer $Q$ using FIT2D (Hammersley et al. 2006). The program xPDFsuite (Yang et al. 2014) was used to correct and normalize the diffraction data, and then Fourier transform them to obtain the experimental PDF, $G(r)$, according to

$$
G(r)=\frac{2}{\pi} \int_{\min }^{Q_{\max }} Q[S(Q)-1] \sin Q r d Q
$$

where $S(Q)$ are the properly corrected and normalized powder diffraction intensities measured from $Q_{\min }$ to $Q_{\max }$, in this case 0.6 and $20.0 \AA^{-1}$, respectively. The reduced total scattering structure function is given by $F(Q)=Q[S(Q)-1]$. The nickel standard measurement was also used to determine the instrument resolution effects on the resulting PDFs. An FCC structure was fit to the PDF using the program PDFgui (Farrow et al. 2007)
, and the resulting resolution parameters were determined $\left(Q_{\text {damp }}=0.0384 \AA^{-1}\right.$ and $Q_{\text {broad }}=$ $0.0165 \AA^{-1}$ ) and fixed in subsequent refinements. For a known structure model, the PDF is simulated by

$$
\begin{aligned}
& G(r)=4 \pi r \gamma_{0}(r)\left[\rho(r)-\rho_{0}\right] \\
& \rho(r)=\frac{1}{4 \pi r^{2} N} \sum_{j} \sum_{j^{1} i} \frac{f_{i}^{*} f_{j}}{\langle f\rangle^{2}} \delta\left(r-r_{i j}\right)
\end{aligned}
$$

where $\rho_{0}$ is the atomic number density, $\rho(r)$ is the atomic pair density, which is the mean weighted density of neighbour atoms at distance $r$ from an atom at the origin, and $\gamma_{0}(r)$ is function which modulates the signal based on the shape and size of the coherently scattering domains in the material (Farrow and Billinge 2009). The sums run over all atoms in the unit cell with periodic boundary conditions applied. $f_{i}$ and $f_{j}$ are the scattering factors for atoms $i$ and $j,\langle f\rangle$ is the average scattering factor, and $r_{i j}$ is the distance between atoms $i$ and $j$. In this study, this method was applied using PDFgui, where models for separate phases were co-refined by allowing parameters including the scale of the phase, lattice parameters, thermal displacement parameters, a correction term for correlated motion, and the diameter of a finite spherical domain defining $\gamma_{0}(r)$ (Farrow and Billinge 2009), to refine.

\section{Fabrication of working electrodes}

The working electrodes were fabricated by mixing biogas derived activated carbon $(4 \mathrm{mg})$, conducting carbon $(0.5 \mathrm{mg})$, and polyvinylidene fluoride (PVDF) $(0.5 \mathrm{mg})$. The mixtures were pressed onto nickel foam of 1 $\mathrm{cm} \times 1 \mathrm{~cm}$.

\section{Electrochemical measurements}

Electrochemical measurements were performed using computer controlled AUTOLAB PGSTAT204 (Metrohm). Platinum was used as the counter electrode while silver/silver chloride $(\mathrm{Ag} / \mathrm{AgCl})$ in a $\mathrm{KCl}$ solution was used 
as reference electrode. All electrochemical measurements were performed at room temperature in $6 \mathrm{M} \mathrm{KOH}$ in a three-electrode cell setup. The cyclic voltammetry was performed between a potential range of -1 to 0 $\mathrm{V}$ at scan rates of $5,10,20,30,50$, and $150 \mathrm{~m}$ $\mathrm{V} \mathrm{s}^{-1}$. The electrochemical impedance spectroscopy (EIS) was performed using FRA32 Module in the frequency range of 1 $\mathrm{mHz}$ to $0.1 \mathrm{MHz}$ with an AC amplitude of 10 $\mathrm{mV}$ at open circuit voltage. The specific capacitances $(C)$ of different electrodes at different scan rates $\left(\mathrm{mV} \mathrm{s}^{-1}\right)$ in the $\mathrm{CV}$ measurements were calculated using Equation 4.

$$
C=\frac{1}{2 m v\left(V_{2}-V_{1}\right)} \int_{V 1}^{V 2} i(V) d V
$$

\section{Results and Discussion}

\section{Physical characterization of carbon} materials

Figure 1 shows the SEM micrographs for the synthesized samples. The unactivated sample exhibited irregular, small particles, Figure 1A with small pores. BC-0-4 had an irregular pore structure from the $\mathrm{KOH}$ treatment, Figure 1B. BC-4-0 sample showed irregular particles, Figure 1C. On the other hand, sample BC-2-2 showed cylinder-like porous structures, Figure 1D. Figure 1E shows disintegration of the cylinder-like pore structure when more $\mathrm{KOH}$ was used. When the ratio of $\mathrm{ZnCl}_{2}$ to $\mathrm{KOH}$ was changed to $3: 1$, no obvious change in morphology was observed. Simultaneous use of $\mathrm{KOH}$ and $\mathrm{ZnCl}_{2}$ led to drastic morphology change. It was found that as the ratio of $\mathrm{ZnCl}_{2}$ increased, the porous structure broke down (Figure 1D, E, and F). From SEM micrographs (Figure 1B-F), one can see that these samples had open pores on the surface which could facilitate the buffering of ions thus providing short space for the electrolyte ions diffusion (Xing et al.2016).

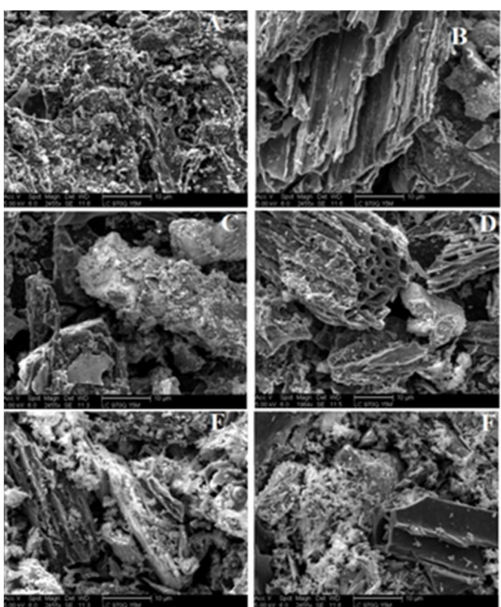

Figure 1: SEM micrographs of samples

(A) BC-0-0, (B) BC-0-4, (C) BC-4-0,

(D) BC-2-2, (E) BC-1-3 and (F) BC-3-1.

The effects of activating agents on the pore structure and surface properties of the synthesized porous carbon were investigated using $\mathrm{N}_{2}$ adsorption and desorption techniques. Figure 2A shows nitrogen and desorption isotherms of BC-4-0, BC-0-4, BC-3-1, BC-1-3, and $\mathrm{BC}-2-2$ samples. All samples exhibited a type IV isotherm with a well-pronounced $\mathrm{H} 4$ type of hysteresis loop, indicating that the mesopores were well developed. The isotherms also demonstrate that there was a complete filling of the mesopores due to the capillary condensation, which starts at $\frac{p}{p_{0}}=0.5$ to approximately $\frac{p}{p_{0}}=1$. The adsorption/ desorption curves of $\mathrm{BC}-0-3$ and $\mathrm{BC}-2-2$ almost coincide, implying that their adsorption capacities are the same. Figures $2 \mathrm{~B}$ and $2 \mathrm{C}$ show nitrogen adsorption and desorption isotherms for BC-4-0 and BC-3-1, respectively. The isotherms for these two samples did not close at low relative pressure $(<0.1)$, indicating that the sorption was not complete. 

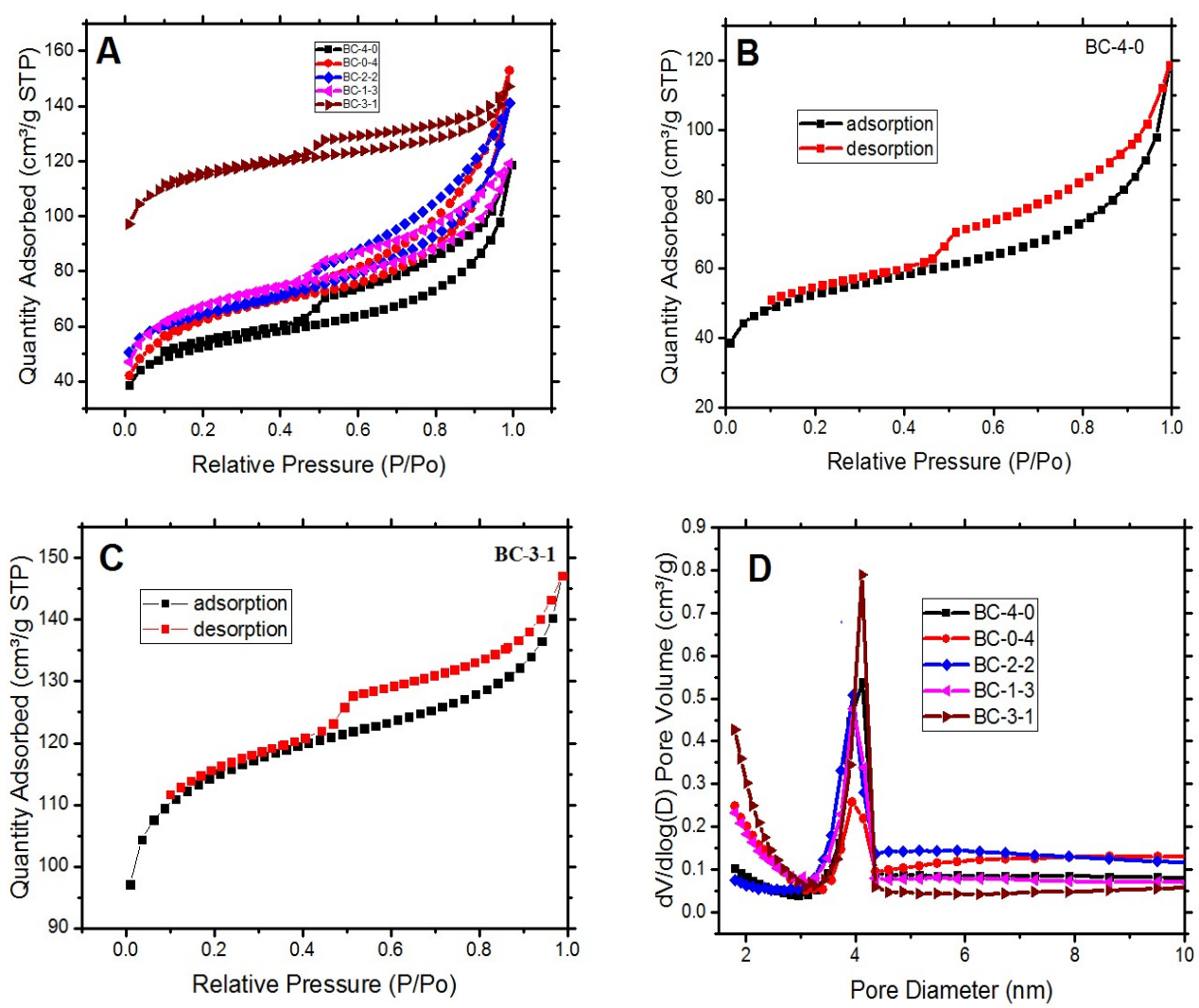

Figure 2: (A) Nitrogen adsorption and desorption isotherms of all samples, (B) Nitrogen adsorption and desorption isotherms of BC-4-0, (C) Nitrogen adsorption and desorption isotherms of $\mathrm{BC}-3-1$ and (D) Pore size distribution calculated using $\mathrm{BJH}$ method.

The pore distributions as calculated from the desorption branch are shown in Figure 2D. All the samples had pores approximately $4 \mathrm{~nm}$ in size, which is in the mesopore range. Notably, the highest degree of mesoporosity was obtained when $\mathrm{KOH}$ and $\mathrm{ZnCl}_{2}$ were used simultaneously. The magnitude of the peak at 4 nm was observed in the order of $\mathrm{BC}-3-1>\mathrm{BC}$ 4-0 > BC-2-2 > BC-1-3 > BC-0-4 implying that $\mathrm{KOH}$ has little influence on the pore size. These mesopores are very important in facilitating charge storage as well as charge transfer in supercapacitors. In Figure 2B, the complete lower limit of the pore size is not shown in the distribution, demonstrating the existence of micropores.

Table 1 shows the pore textures of the synthesized porous carbon materials. It can be seen that the $\mathrm{ZnCl}_{2}$ itself (BC-4-0) had no significant effect on the BET surface area (171 $\mathrm{m}^{2} \mathrm{~g}^{-1}$ ). When $\mathrm{KOH}$ alone (BC-0-4) was used, $206 \mathrm{~m}^{2} \mathrm{~g}^{-1}$ surface area was achieved. BC-0-4 and BC-2-2 exhibited approximately the same BET surface area $\left(206 \mathrm{~m}^{2} \mathrm{~g}^{-1}\right)$. These results are consistent with those presented in Figure 3A. BC-3-1 exhibited the highest BET surface area $\left(356 \mathrm{~m}^{2} \mathrm{~g}^{-1}\right)$ signifying the importance of both $\mathrm{KOH}$ and $\mathrm{ZnCl}_{2}$ in activating the 
materials. The micropore surface area followed the same trend as that of the BET surface area. Though there is no defined trend of mesopore content as the $\mathrm{ZnCl}_{2}$ and $\mathrm{KOH}$ ratios were altered, BC-0-4 attained $85 \%$ of mesopore content, followed by BC-3-1 (78\%). The high mesopore content for $\mathrm{BC}-0-4$ may be due to the high volatilisation of the organics by $\mathrm{KOH}$. The average pore sizes for BC-4-0, BC-0-4 and BC-2-2 samples were comparatively equal, meaning that the synthesis routes did not change the sizes of the pores. On the other hand, BC-3-1 and BC-1-3 had lower average pore sizes compared to other three samples.

Table 1: Pore texture and surface area of the $\mathrm{BC}$ mesoporous carbon samples

\begin{tabular}{|c|c|c|c|c|c|c|c|c|}
\hline Sample & $\begin{array}{l}{ }^{1} \mathrm{~S}_{\mathrm{BET}} \\
\left(\mathrm{m}^{2} \mathrm{~g}^{-1}\right)\end{array}$ & $\begin{array}{l}{ }^{2} \mathrm{~S}_{\text {micro }} \\
\left(\mathrm{m}^{2} \mathrm{~g}^{-1}\right)\end{array}$ & $\begin{array}{l}{ }^{3} \mathrm{~S}_{\text {meso }} \\
\left(\mathrm{m}^{2} \mathrm{~g}^{-1}\right)\end{array}$ & $\begin{array}{l}{ }^{4} V_{\text {micro }} \\
\left(\mathrm{cm}^{3} \mathrm{~g}^{-1}\right)\end{array}$ & $\begin{array}{l}{ }^{5} V_{\text {meso }} \\
\left(\mathrm{cm}^{3} \mathrm{~g}^{-1}\right)\end{array}$ & $\begin{array}{l}{ }^{6} V_{T} \\
\left(\mathrm{~cm}^{3} \mathrm{~g}^{-1}\right)\end{array}$ & $\% \mathrm{~V}_{\text {meso }}$ & $\begin{array}{l}{ }^{7} \mathrm{D}_{\text {Average }} \\
(\mathrm{nm})\end{array}$ \\
\hline BC-4-0 & 171 & 120 & 51 & 0.06 & 0.12 & 0.18 & 67 & 6.1 \\
\hline BC- $0-4$ & 206 & 140 & 66 & 0.03 & 0.18 & 0.21 & 85 & 6.1 \\
\hline BC-2-2 & 206 & 137 & 68 & 0.07 & 0.15 & 0.22 & 68 & 6.3 \\
\hline BC-1-3 & 220 & 176 & 44 & 0.09 & 0.09 & 0.18 & 50 & 4.4 \\
\hline BC-3-1 & 356 & 231 & 125 & 0.05 & 0.18 & 0.23 & 78 & 4.1 \\
\hline
\end{tabular}

1-BET surface area, 2-Micropore surface area, 3-Mesopore surface area, 4-Micropore volume, 5Mesopore volume, 6- Total pore volume and 7-Average pore size.

Pair distribution function analysis (PDF) of the synthesized materials

PDF analysis was used to determine the range of order and phases present in the samples. The reduced total scattering structure functions measured and the resulting PDFs from Fourier transformation are shown in Figures $3 \mathrm{~A}$ and $3 \mathrm{~B}$, respectively. It is worth noting that the curves have been individually rescaled for visual comparison, and the scaling constant is given above the curves in the plot. The structure functions in Figure 3A have been ordered based on apparent relative crystallinity, qualitatively observed by the presence of sharp
Bragg peaks in the scattering pattern. The same Bragg reflections were observed in the samples activated using $\mathrm{ZnCl}_{2}$ and $\mathrm{KOH}, \mathrm{BC}-3-1, \mathrm{BC}-$ 2-2, and $\mathrm{BC}-3-1$. In the case of $\mathrm{BC}-2-2$, some additional sharp Bragg peaks were also present indicating the presence of another crystalline phase. More diffuse scattering was present for the untreated sample, BC-0-0, though different and less intense, Bragg reflections were still present; indicating the presence of both disordered and ordered phases. The BC-4-0 and $\mathrm{BC}-0-4$ structure functions appear to be the most disordered, though, they also have minority Bragg scattering components. 

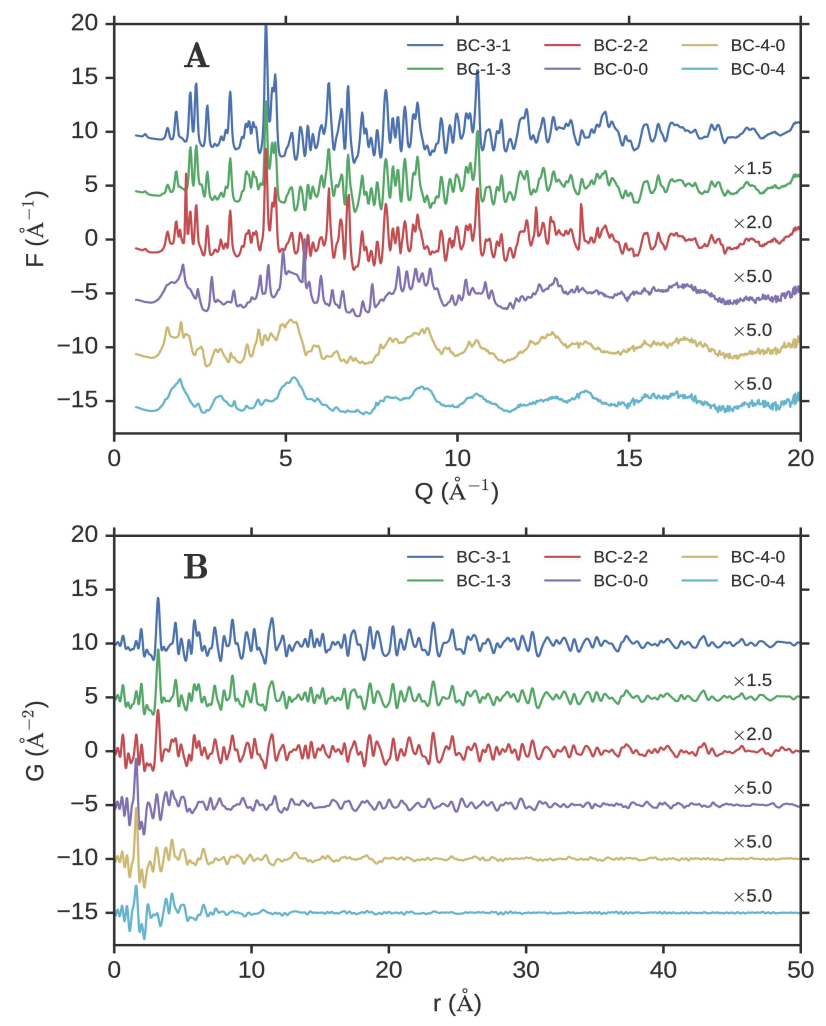

Figure 3: (A) Reduced total scattering structure functions, $F(Q)$, for the untreated and treated samples. (B) The resulting PDFs after Fourier transformation of the respective structure function in A.

The Pearson product-moment correlation coefficient was calculated between the PDFs, over different $r$-ranges, in order to compare the likeness between curves for the different samples. The resulting values can be seen in Table 2. The first number is for a range of 1.0 $10.0 \AA$ and the numbers in parentheses correspond to a range of $10.0-50.0 \AA$. Large numbers are bold representing a high degree of similarity between the structures over the given range. The results confirm the expectations from before. The samples activated with both $\mathrm{ZnCl}_{2}$ and $\mathrm{KOH}$ were the most crystalline as indicated by the presence of high amplitude, sharp peaks present out to high distances, and all had highly similar local and long range structures present. On the other hand, the unactivated sample, and the samples activated using only $\mathrm{ZnCl}_{2}$ or $\mathrm{KOH}$, were all different from the co-activated samples. These three samples had similar local structures, but were remarkably different over long ranges. The long-long range signals in $\mathrm{BC}-0-0$ and $\mathrm{BC}-4-0$ extended to high distances but had an overall smaller peak to trough amplitude indicating that long range ordered structures were present but different than that in the co-activated samples, and at lower concentrations. BC-0-4 appeared to be the most disordered sample. 
Tanz. J. Sci. Vol. 47(4) 2021

Table 21: Pearson product-moment correlation coefficients calculated from the experimental PDFs of the different samples

\begin{tabular}{lllllll}
\hline Sample & BC-3-1 & BC-1-3 & BC-2-2 & BC-0-0 & BC-4-0 & BC-0-4 \\
\hline BC-3-1 & 1.0 & $\mathbf{0 . 9 6}(\mathbf{0 . 9 7})$ & $\mathbf{0 . 9 0}(\mathbf{0 . 9 3})$ & $0.02(-0.11)$ & $0.12(-0.21)$ & $-0.03(-0.08)$ \\
BC-1-3 & - & 1.0 & $\mathbf{0 . 9 2}(\mathbf{0 . 9 1})$ & $0.11(-0.06)$ & $0.22(-0.12)$ & $0.03(-0.03)$ \\
BC-2-2 & - & - & 1.0 & $0.36(-0.12)$ & $0.47(-0.11)$ & $0.29(-0.01)$ \\
BC-0-0 & - & - & - & 1.0 & $\mathbf{0 . 9 2}(0.04)$ & $\mathbf{0 . 9 0}(0.19)$ \\
BC-4-0 & - & - & - & - & 1.0 & $\mathbf{0 . 8 8}(0.53)$ \\
BC-0-4 & - & - & - & - & - & 1.0 \\
\hline
\end{tabular}

Based on sample compositions from EDX, the PDFs for the co-activated samples were indexed against a wide range of potential structures, and the crystal structure was determined to be consistent with $\mathrm{Zn}_{2} \mathrm{SiO}_{4}$ willemite. Single phase fits of the willemite structure of these sample PDFs are shown in Figure 4. BC-3-1 is very well described by willemite, and it only has some misfit structural features at low- $r$ indicating the presence of some short-range ordered component. The goodness-of-fit for BC-1-3 and BC-2-2 is much worse, and it is clear from the differences that there is likely additional short- and long-range ordered structural components present which are not described by willemite. The differences might be due to the fact that combined activation $\left(\mathrm{ZnCl}_{2}\right.$ and $\left.\mathrm{KOH}\right)$ leave some residues to the samples. Also the amounts of silica might have altered the atomic distances.

To test this, the more disordered samples were analysed to determine the short-range components present in the active materials. A comparison of these samples is made in Figure 5. The first and second nearest neighbour distances for graphite and the $\mathrm{Si}-\mathrm{O}$ distance in silica are labelled, showing that a mixture of these components is present in the samples (Figure 5A). In Figure 5B, it is shown that the unfit components in the crystalline samples likely come from the remaining disordered carbonaceous and siliceous components.

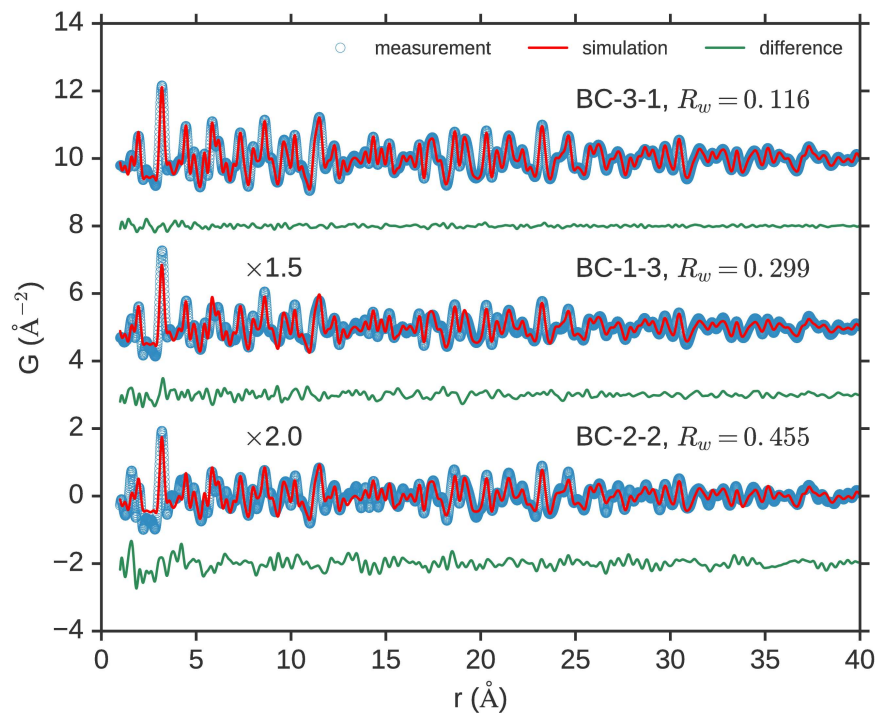

Figure 4: PDFs simulated from refinement of the willemite structure (red) to the measured PDFs (blue), over a range of 1.0-40.0 A. Differences are plotted below in green. 


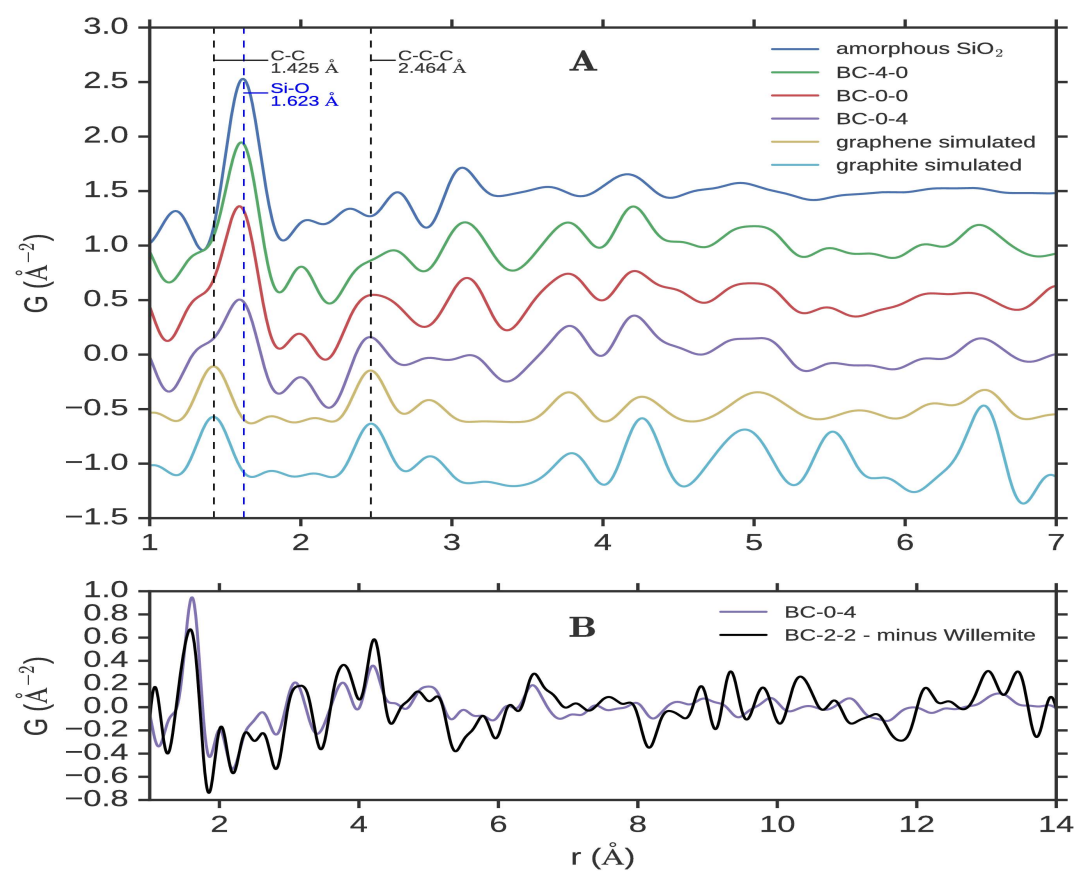

Figure 5: (A) Measured PDFs for BC-4-0, BC-0-0, and BC-0-4 are compared to a PDF measured for amorphous silica, as well as simulated PDFs for both graphite and a single layer of graphene. (B) The PDF measured for BC-0-4 compared to the residual after subtracting willemite from BC-2-2.

All of the disordered samples have a large peak at approximately $1.62 \AA$, which corresponds to the $\mathrm{Si}-\mathrm{O}$ neighbour distance in silica. This agrees well with the high level of $\mathrm{Si}$ present in the samples observed with EDX. There is also a shoulder on the first peak at approximately $1.4 \AA$ which comes from the active carbon phase in the samples. Upon further examination, it is clear that the local structure shows similarities that are intermediate between the local structure of amorphous silica, and the local structure of graphene. This indicates that the carbon components present are likely highly turbostratically disordered layers of graphite which is better for supercapacitor applications. Unfortunately, the long range structures in these three samples were not successfully indexed, which is likely due to some longer range ordered phases consisting of silicon dioxide, or in combination with the other alkali and alkaline earth metal impurities present, observed with EDX. However, the focus was on fitting the local structure of these phases to get an estimate of types of local structuring, as well as the relative phase fractions present.

The carbon component was fitted with a graphite $2 \mathrm{H}$ structure, with $\mathrm{P} 63 / \mathrm{mmc}$ symmetry, with ICSD \#76767. Turbostratic disorder was modelled by allowing separate thermal displacement values in the plane of the layer and out-of-plane between the layers, such that interlayer correlations were significantly broadened out. The silica component was modelled using $\alpha$-Quartz low, with P3231 symmetry, ICSD \#16331. The multiphase fits including the carbon and silica components are shown for all the six samples in Figure 6. The resulting phase fractions are shown in Table 3. 

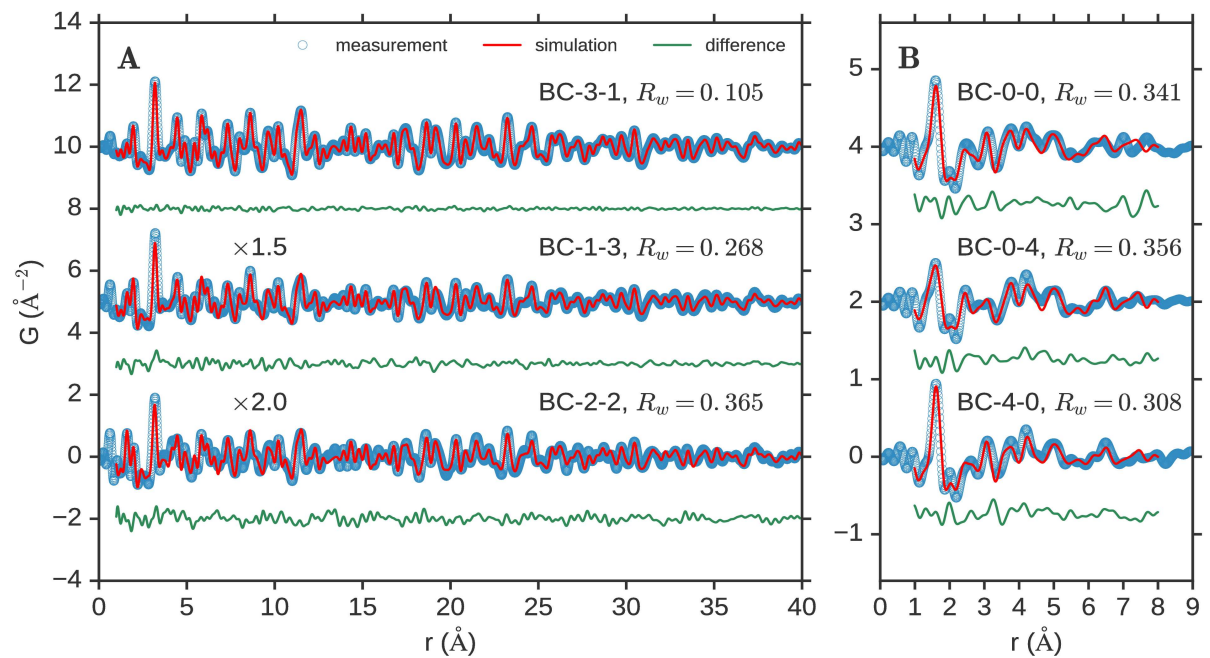

Figure 61: (A) PDFs simulated from three-phase refinement of graphite + quartz + willemite (red) to the measured PDFs (blue), over a range of 1.0-40.0 $\AA$. Differences are plotted below in green. (B) Simulated PDFs (red) from refinement of graphite + quartz over a range of 1.0-8.0 ̊̊.

Table 3: The resulting weight percent and coherence length for the phases fit in the models described

\begin{tabular}{cllllll}
\hline & BC-0-0 & BC-4-0 & BC-3-1 & BC-2-2 & BC-1-3 & BC-0-4 \\
\hline Carbon $\mathrm{wt}^{2} \%$ & $0.31^{\mathrm{a}}$ & 0.22 & 0.16 & 0.52 & 0.44 & 0.44 \\
$\mathrm{~d}_{\mathrm{c}}(\AA)$ & $-\mathrm{b}$ & - & 10 & 50 & 21 & - \\
Quartz wt $\%$ & 0.69 & 0.78 & 0.30 & 0.33 & 0.47 & 0.56 \\
$\mathrm{~d}_{\mathrm{c}}(\AA)$ & 6 & 6 & 7 & 8 & 7 & 7 \\
Willemite $\mathrm{wt} \%$ & - & - & 0.54 & 0.15 & 0.09 & - \\
$\mathrm{d}_{\mathrm{c}}(\AA)$ & - & - & 218 & 343 & 186 & - \\
Range $(\AA)$ & $1.0-8.0$ & $1.0-8.0$ & $1.0-40.0$ & $1.0-40.0$ & $1.0-40.0$ & $1.0-8.0$ \\
$\mathrm{R}_{\mathrm{w}}$ & 0.341 & 0.308 & 0.105 & 0.365 & 0.268 & 0.356 \\
\hline
\end{tabular}

${ }^{a}$ As the models are only rough approximations; phase fractions should only be considered relatively.

${ }^{\mathrm{b}}$ The actual coherence lengths for the carbon phase are likely much smaller than the values achieved through the refinement, as phases may try to fit peaks from other structural components or even noise when the signal is very weak.

Electrochemical performance of the synthesized porous materials

Figure 7 shows the intrinsic electrochemical characteristics of the synthesized porous materials using cyclic voltammograms at scan rates of 5 to $150 \mathrm{mV} \mathrm{s}^{-1}$. It can be seen that the $\mathrm{CV}$ curves maintained the rectangular shapes even at high scan rates, indicating good capacitive behaviour of the materials. In Figure
$7 \mathrm{~A}$, the unactivated sample (BC-0-0) shows CV curves dramatically distorted from the ideal rectangular shape. This is indicative of poor capacitive behaviour resulting probably from low surface area and micropore and mesopore content.

Figure 7B-E shows charge and discharge profiles/curves that are nearly rectangular in shape at all the scan rates suggesting 
remarkable capacitive behaviour. However, as shown in Figure 7E-F, for BC-3-1, BC-1-3, samples, there are some humps on the $\mathrm{CV}$ curves that might be attributed to the faradaic reactions associated with the redox couples originating from crystalline inorganic phases in the BC-3-1 and BC-1-3 samples as also confirmed by PDF results. Furthermore, the
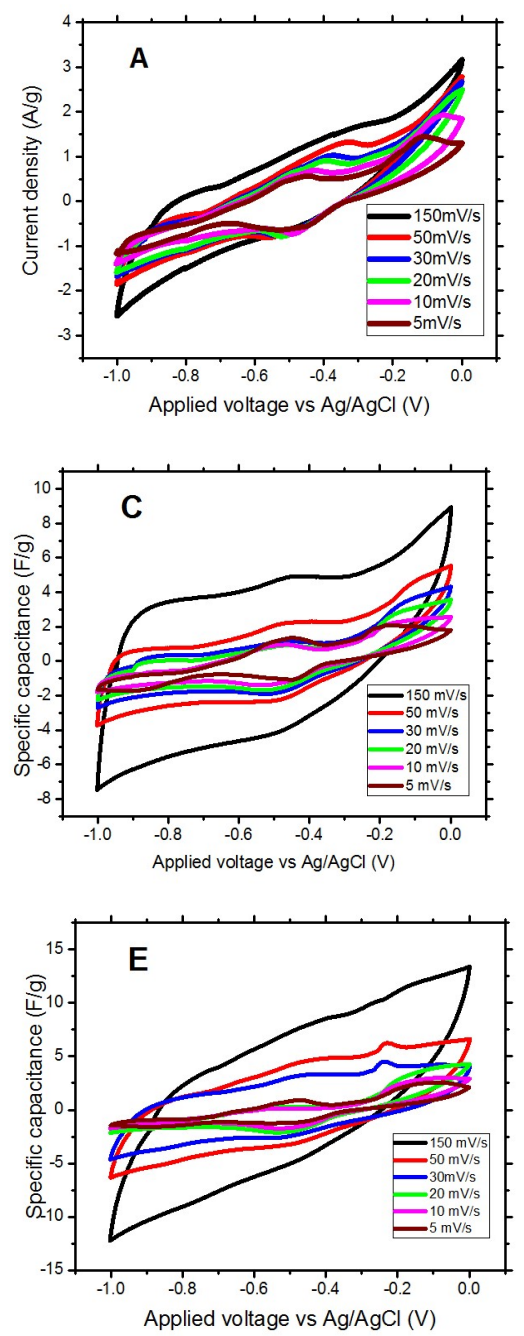

$\mathrm{CV}$ voltammograms of the $\mathrm{BC}-3-1$ sample show marked deviation from the ideal rectangular shape compared to the other samples. This was probably due to the significant amount of residual crystalline $\mathrm{ZnO}$ and other crystalline inorganic derivatives of $\mathrm{Zn}$, for instance, $\mathrm{Zn}_{2} \mathrm{SiO}_{4}$ willemite that greatly reduce the capacitive behaviour
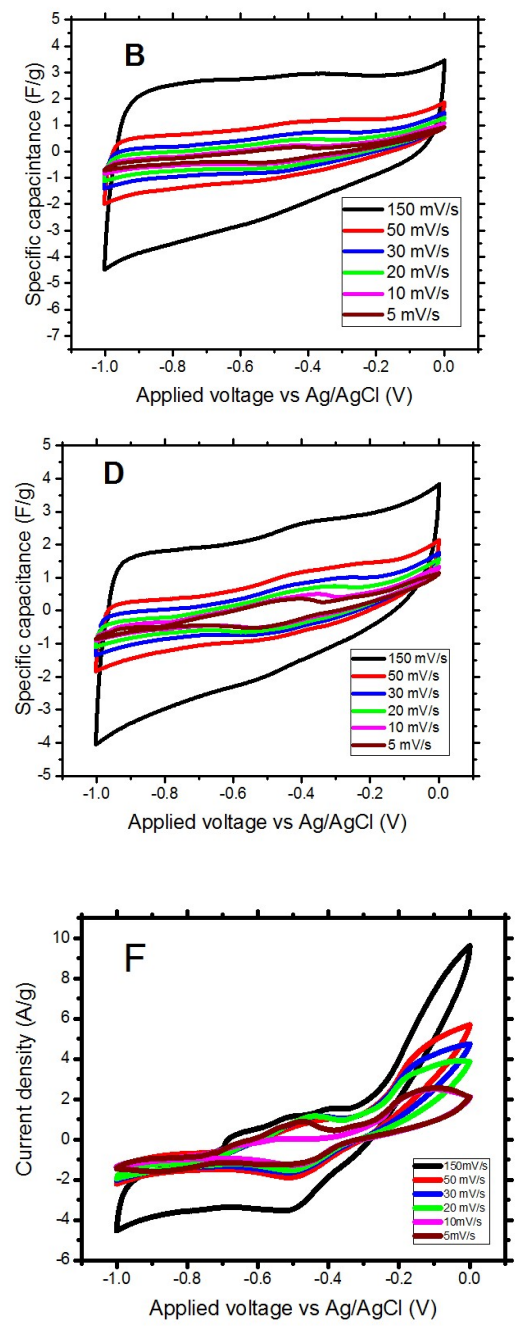

Figure 7: Cyclic voltammograms of the synthesized electrodes (A) B-0-0, (B) B-4-0, (C) B-0-4, (D) B-2-2, (E) B-1-3 and (F) B-3-1. 
The specific capacitances as calculated from the CV graphs are shown in Figure 8A. It was observed that there was no obvious trend between the $\mathrm{ZnCl}_{2}: \mathrm{KOH}$ ratio and the specific capacitance. At scan rate of $5 \mathrm{mVs}^{-1}$, the unactivated sample exhibited a specific capacitance of $126 \mathrm{~F} \mathrm{~g} \mathrm{~g}^{-1}$, while the capacitance increased to $143,202,161,210$ and $216 \mathrm{~F} \mathrm{~g}^{-1}$ for $\mathrm{BC}-0-4, \mathrm{BC}-4-0, \mathrm{BC}-2-2, \mathrm{BC}-1-3$ and $\mathrm{BC}-$ $3-1$, respectively. The obtained specific capacitance is lower than those obtained for phenolic resin based carbon fibers $\left(288 \mathrm{~F} \mathrm{~g}^{-1}\right.$ ) (Ma et al. 2017). From Figure 8A, it is clear that the combining of both the $\mathrm{KOH}$ and $\mathrm{ZnCl}_{2}$ during activation is more suitable for supercapacitors materials.

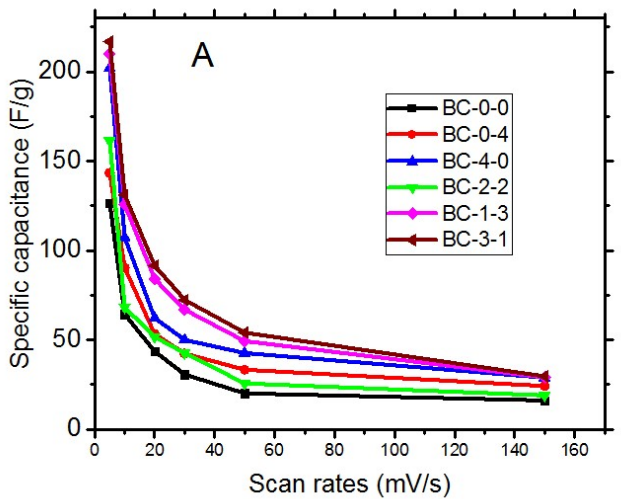

Figure $8 \mathrm{~b}$ shows the electrochemical impedance spectroscopy (EIS) of the fabricated electrodes. The Nyquist plots demonstrate that all samples had high conductivity since the total holmic resistances are low (the intercept with real $\mathrm{Z}$ high frequencies). The series resistance is lower than $1 \Omega$ for all samples, implying that the electrodes had high ionic conductivity (see the inset of Figure 8B). Moreover, BC-3-1 exhibited lower internal resistance than other samples. This might be the reason why BC-3-1 exhibited high specific capacitance. The Warburg diffusion, which is represented by the vertical nature of the graphs at low frequency, shows that $\mathrm{BC}-0-4$ had more capacitive behaviour than the other electrodes.

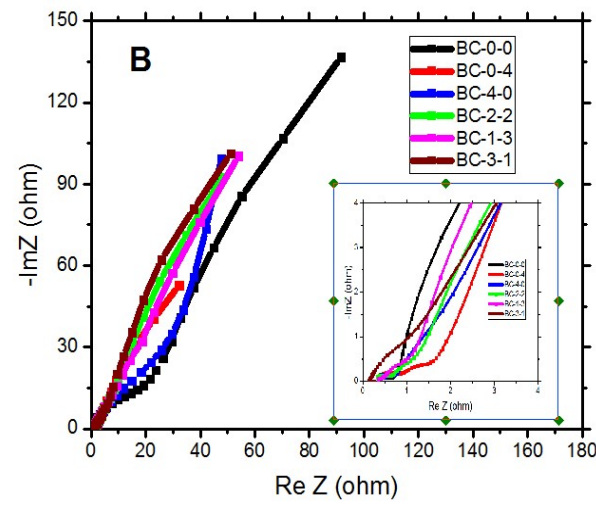

Figure 8: (A) Specific capacitance of synthesized porous carbon and (B) Nyquist plots of synthesized electrodes.

\section{Conclusion}

$\mathrm{ZnCl}_{2}$ as a molten salt and $\mathrm{KOH}$ as an activating agent have been used to synthesize mesoporous carbon from biogas slurry. The ratios between $\mathrm{ZnCl}_{2}$ and $\mathrm{KOH}$ were varied from $0: 4,1: 3,2: 2,3: 1$ to $4: 0$. The samples were activated at $700{ }^{\circ} \mathrm{C}$ for $1 \mathrm{~h}$ under nitrogen flow. Nitrogen sorption studies revealed that mesoporous carbon materials were successfully synthesized. The BC-3-1 showed the highest uptake of nitrogen as well as the highest specific surface area $\left(356 \mathrm{~m}^{2} \mathrm{~g}^{-1}\right)$. The short and long range structures of the samples as studied using pair distribution function (PDF) showed significant amount of siliceous components in the form of crystalline willemite in samples activated with both $\mathrm{ZnCl}_{2}$ and $\mathrm{KOH}$, and amorphous or medium range ordered silica. The electrochemical studies in three-electrode cell system revealed high specific capacitance of $216 \mathrm{~F} \mathrm{~g} \mathrm{~g}^{-1}$ exhibited by the sample with $\mathrm{ZnCl}_{2}: \mathrm{KOH}$ mass ratio of 3:1:at scan rate of $5 \mathrm{mV} \mathrm{s}^{-1}$, implying that both $\mathrm{ZnCl}_{2}$ and $\mathrm{KOH}$ had significant impact in development of the materials. However, it is important to devise a mechanism for removing silica content from the samples. 
Conflict of interest: Authors have no conflicts of interest to disclose.

\section{Acknowledgements}

This research used beamline 28-ID-2 of the National Synchrotron Light Source II, a US Department of Energy (DOE) Office of Science User Facility operated for the DOE Office of Science by Brookhaven National Laboratory under Contract No. DESC0012704. We are also grateful to Hanyang University (South Korea) for BET analysis.

\section{References}

Baig MM and Gul IH 2021 Conversion of wheat husk to high surface area activated carbon for energy storage in highperformance supercapacitors. Biomass Bioenergy 144: 105909.

Cao Q, Zhang Y, Chen J, Zhu M, Yang C, Guo $\mathrm{H}$, Song Y, Li Y, and Zhou J 2020 Electrospun biomass based carbon nanofibers as high-performance supercapacitors. Ind. Crops Prod. 148: 112181.

Castro-Gutiérrez J, Díez N, Sevilla M, Izquierdo MT, Celzard A, and Fierro V 2021 Model carbon materials derived from tannin to assess the importance of pore connectivity in supercapacitors. Renewable and Sustainable Energy Reviews 151: 111600.

Chupas PJ, Qiu X, Hanson JC, Lee PL, Grey $\mathrm{CP}$ and Billinge SJL 2003 Rapidacquisition pair distribution function (RAPDF) analysis. J. Appl. Cryst. 36(6): 13421347.

Elisadiki J, Jande YAC, Machunda RL, and Kibona TE 2019 Porous carbon derived from Artocarpus heterophyllus peels for capacitive deionization electrodes. Carbon 147: 582-593.

Enock TK, King'ondu CK, Pogrebnoi A, and Jande YAC 2017 Biogas-slurry derived mesoporous carbon for supercapacitor applications. Materials Today Energy 5: 126-137.

Farrow CL and Billinge SJL 2009 Relationship between the atomic pair distribution function and small-angle scattering: implications for modeling of nanoparticles. Acta Cryst. A 65(3): 232-239.

Farrow CL, Juhas P, Liu JW, Bryndin D, Božin ES, Bloch J, Proffen T, and Billinge SJL 2007 PDFfit2 and PDFgui: computer programs for studying nanostructure in crystals. J. Phys.: Condens. Matter 19(33): 335219.

Hammersley AP, Svensson SO, Hanfland M, Fitch AN, and Hausermann D 2006 Twodimensional detector software: From real detector to idealised image or two-theta scan. High Pressure Res. Int. J. 14(4-6): 235-248.

$\mathrm{Hu}$ SC, Cheng J, Wang WP, Sun GT, Hu L Le, Zhu MQ, and Huang XH 2021 Structural changes and electrochemical properties of lacquer wood activated carbon prepared by phosphoric acid-chemical activation for supercapacitor applications. Renew. Energy 177: 82-94.

Kibona TE 2019 Highly microstructured porous carbon derived from vitex mombassae for high specific capacitance supercapacitors. SN Appl. Sci. 1(9): 1-11.

Lin H, Liu Y, Chang Z, Yan S, Liu S, and Han S 2020 A new method of synthesizing hemicellulose-derived porous activated carbon for high-performance supercapacitors. Microporous Mesoporous Mater. 292: 109707.

Ma C, Song Y, Shi J, Zhang D, Zhai X, Zhong M, Guo Q, and Liu L 2013 Preparation and one-step activation of microporous carbon nanofibers for use as supercapacitor electrodes. Carbon 51(1): 290-300.

Ma C, Wang R, Xie Z, Zhang H, Li Z, and Shi J 2017 Preparation and molten salt-assisted $\mathrm{KOH}$ activation of porous carbon nanofibers for use as supercapacitor electrodes. J. Porous Mater. 24(6): 14371445.

Shang H, Lu Y, Zhao F, Chao C, Zhang B and Zhang H 2015 Preparing high surface area porous carbon from biomass by carbonization in a molten salt medium. $R S C$ 
Advances 5(92): 75728-75734.

Wang J, Zhang X, Li Z, Ma Y, and Ma L 2020

Recent progress of biomass-derived carbon materials for supercapacitors. J. Power Sources 451: 227794.

Xing B, Huang G, Chen L, Guo H, Zhang C, Xie W, and Chen Z 2016 Microwave synthesis of hierarchically porous activated carbon from lignite for high performance supercapacitors. J. Porous Mater. 23(1): 67-73.

Yang X, Juhas P, Farrow CL, and Billinge SJL 2014 xPDFsuite: an end-to-end software solution for high throughput pair distribution function transformation, visualization and analysis. J. Appl. Cryst. arXiv preprint arXiv: 1402.3163 . 